\title{
Interferometer Based on a D-shape Chaotic Optical Fiber for Measurement of Multiparameters
}

\author{
Susana SILVA ${ }^{1}$, Luís COELHO ${ }^{1}$, Philippe ROY ${ }^{2}$, and Orlando FRAZÃO ${ }^{*}$ \\ ${ }^{1}$ INESC Porto, Rua do Campo Alegre 687, 4169-007 Porto, Portugal and Dept. de Física e Astronomia da Faculdade de \\ Ciências da Universidade do Porto, Rua do Campo Alegre 687, 4169-007 Porto, Portugal \\ ${ }^{2}$ Xlim UMR 6172 CNRS-University of Limoges, 123 Avenue Albert Thomas, 87060 Limoges Cedex, France \\ ${ }^{*}$ Corresponding author: Orlando FRAZÃO $\quad$ E-mail: ofrazao@inescporto.pt
}

\begin{abstract}
An interferometer based on a D-shape chaotic optical fiber for measurement of multiparameters was proposed. The sensing structure relied on a D-shape fiber section spliced in between two singlemode fibers and interrogated in transmission. The optical spectrum was composed by multiple interference loss peaks, which were sensitive to the refractive index, temperature and strain - maximum sensitivities of $95.2 \mathrm{~nm} / \mathrm{RIU}, 10.5 \mathrm{pm} /{ }^{\circ} \mathrm{C}$ and $-3.51 \mathrm{pm} / \mu \varepsilon$, respectively, could be achieved.
\end{abstract}

Keywords: Optical fiber sensors, interferometer, chaotic fiber

Citation: Susana SILVA, Luís COELHO, Philippe ROY, and Orlando FRAZÃO, "Interferometer Based on a D-shape Chaotic Optical Fiber for Measurement of Multiparameters," Photonic Sensors, DOI: 10.1007/s13320-012-0078-z.

\section{Introduction}

Interferometry in optical fibers is easily fabricated, and distinct geometry has already been developed. Although highly sensitive to various types of physical and chemical parameters, the cross sensitivity to temperature has been always an issue. Many different types of interferometric techniques have been developed for simultaneous measurement of multiparameters. Michelson or Mach Zender interferometers usually require some meters of the fiber to enhance sensitivity $[1,2]$. However, using specific optical devices such as long period grating structures, it is possible to obtain more attractive geometry within some centimeters of fiber [3]. One of the most frequently used techniques is the fiber optic Fabry-Perot interferometer. In this case, cavity lengths with few micrometers are easily obtained [4].
Also, multimodal interference-based fiber devices have been an active area of research. A simple technique is to generate multimode interference among high-order guided modes of a step index multimode fiber spliced in between two single mode fibers [5]. In other hand, intermodal interference using high birefringence fibers has shown high potential to discriminate different physical parameters [6]. More recently, multimode optical fibers with a noncircular cross section have shown great relevance in the research field of wave chaos [7, 8]. Theoretical and experimental studies of double-clad fiber amplifiers have been presented. The chaotic propagation on the inner cladding with a noncircular cross section is used to improve the pumping process $[9,10]$. D-shape optical fibers are multimode chaotic fiber structures with distinct and attractive features that could be suitable for sensing 
applications.

In this work, multimodal interference using a multimode silica fiber with a D-shaped cross section is proposed. An interferometer is obtained by splicing the multimode D-shape chaotic fiber in between two single mode fibers and interrogated in transmission. The spectral response presents multiple interference loss peaks that are sensitive to multiparameters. The interferometer is characterized to refractive index, temperature and strain.

\section{Experimental results}

To perform the experiment, the sensing head structure shown in Fig. 1(a) was implemented. It consisted of a step-index multimode D-shape fiber section spliced in between two SMF-28 singlemode fibers and interrogated in transmission. In this approach, a supercontinuum broadband source with a bandwidth of $500 \mathrm{~nm}$ and an optical spectrum analyzer as the interrogation unit were used. The detail of the D-shape fiber being illuminated by the optical source is also depicted in Fig. 1(a).

The multimode D-shape fiber is made of pure silica, with a refractive index of 1.4435 , and has an estimated diameter $d$ of $195 \mu \mathrm{m}$ and a length $L$ of $9 \mathrm{~cm}$. Figure 1(b) shows the cross section of the fiber used where one can observe the asymmetry of the structure. It has been proven for a multimode optical fiber with a noncircular cross section that the transverse motion of rays was chaotic [11]. Therefore, the interference spectrum of the proposed sensing structure would be the result of multiple wave interference (between a large number of different modes) as it is observed in Fig. 2. The spectral response showed multiple interference loss peaks originated by the spatial chaotic behavior of the D-shape fiber which, in principle, would be sensitive to different physical parameters, viz. refractive index (RI), temperature, and strain.

To study the behavior of the sensing head structure to RI variations, three consecutive loss peaks were used $-\lambda_{1}, \lambda_{2}$ and $\lambda_{3}-$ centered at $1456 \mathrm{~nm}, 1473 \mathrm{~nm}$ and $1492 \mathrm{~nm}$, respectively. The characterization was performed by placing the sensing structure in contact with liquids with the distinct RI in the range of 1.33 to 1.38 . The wavelength variation with the RI of the surrounding liquid is shown in Fig. 3.

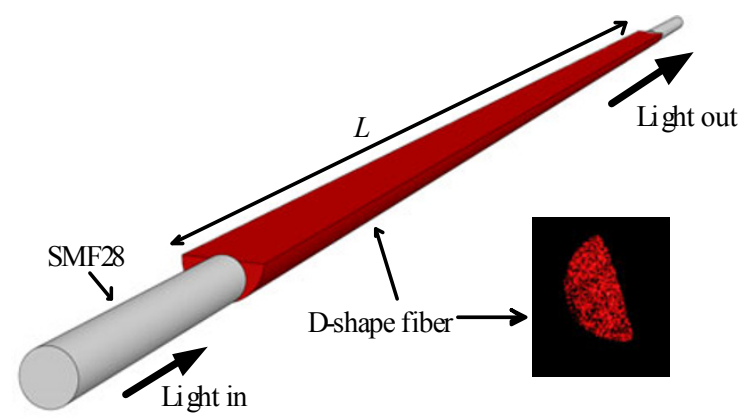

(a)

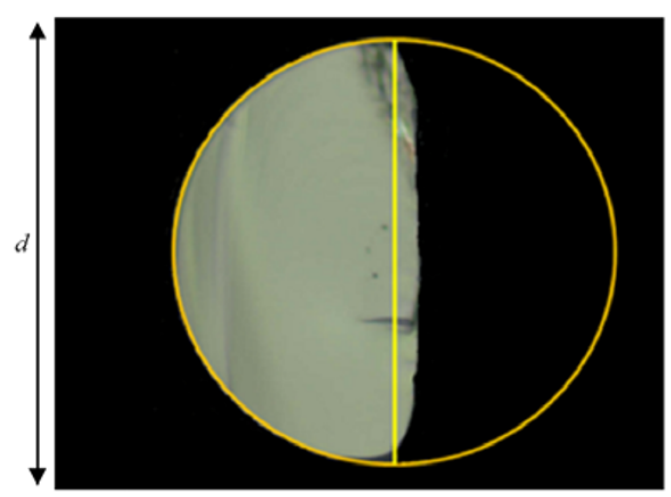

(b)

Fig. 1 Sensing head configuration: (a) schematic of the sensing head structure and detail of the D-shape fiber illuminated by the supercontinuum broadband source and (b) cross section of the D-shape fiber with observed asymmetry of the structure.

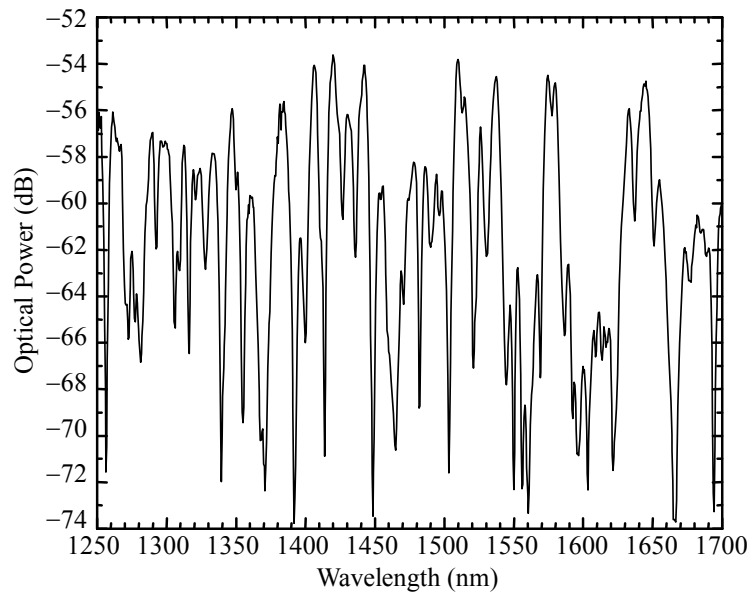

Fig. 2 Refractive index response of the sensing head for the loss bands centered at $\lambda_{1}, \lambda_{2}$ and $\lambda_{3}$. 


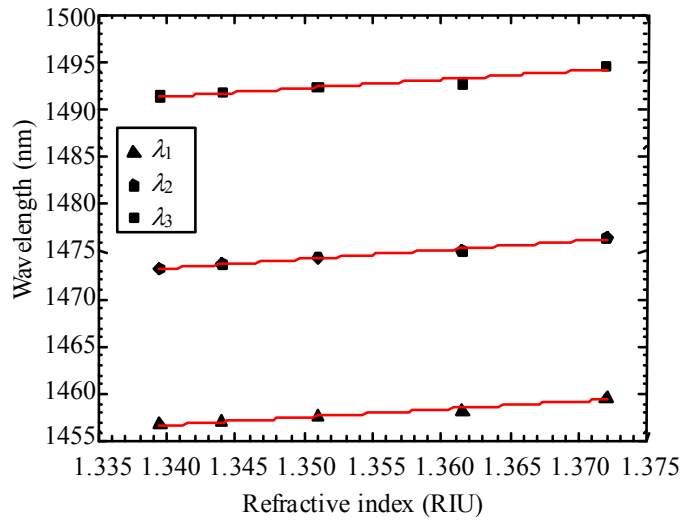

Fig. 3 Refractive index response of the sensing head for the loss bands centered at $\lambda_{1}, \lambda_{2}$ and $\lambda_{3}$.

The D-shape fiber is wavelength dependent on RI changes to the surrounding medium. For the RI range studied, linear responses could be achieved with sensitivities of (83.2 \pm 7.5$) \mathrm{nm} / \mathrm{RIU}$, $(95.2 \pm 6.1) \mathrm{nm} / \mathrm{RIU}$ and $(89.9 \pm 8.5) \mathrm{nm} / \mathrm{RIU}$ for $\lambda_{1}$, $\lambda_{2}$ and $\lambda_{3}$, respectively.

The behavior of the sensing head structure to temperature and strain was accordingly characterized, and in this case, three consecutive loss peaks were used, $-\lambda_{1}, \lambda_{2}$ and $\lambda_{3}$, - centered at $1504 \mathrm{~nm}, 1516 \mathrm{~nm}$ and $1527 \mathrm{~nm}$, respectively.

The response of the sensing head to temperature variations was attained, as shown in Fig. 4. The structure was placed in a tube furnace and submitted to increasing values of temperature in the range of 0 to $350{ }^{\circ} \mathrm{C}$, with $50{ }^{\circ} \mathrm{C}$-step. The loss peaks had similar and linear responses to temperature variations, as shown in Fig. 4, with sensitivity coefficients of $(10.5 \pm 0.6) \mathrm{pm} /{ }^{\circ} \mathrm{C},(10.3 \pm 0.4) \mathrm{pm} /{ }^{\circ} \mathrm{C}$ and $(9.6 \pm 0.4) \mathrm{pm} /{ }^{\circ} \mathrm{C}$ for $\lambda_{1}, \lambda_{2}$ and $\lambda_{3}$, respectively. This was somewhat expected, since the temperature variation did not change the launching conditions between the single mode fiber and the step-index multimode D-shape fiber. The observed behavior of the sensing head to temperature arised mainly from the optical path length variation as a consequence of the D-shape fiber thermal expansion; a slight decrease in sensitivities came from the D-shape fiber refractive index dependence on the wavelength (due to the silica thermo-optic effect) [12].

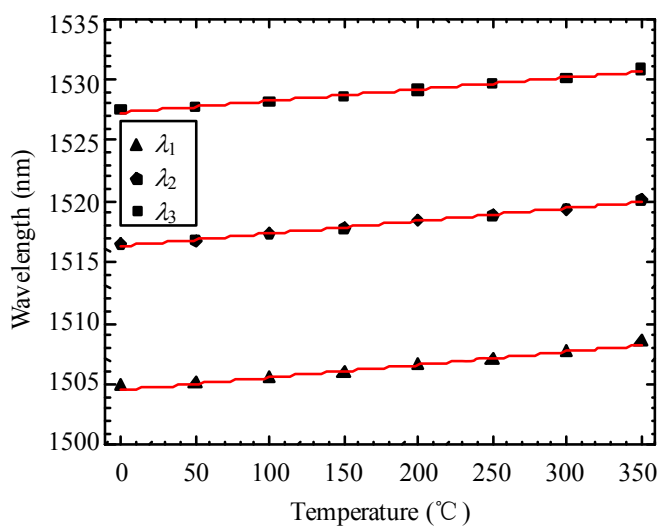

Fig. 4 Temperature response of the sensing head for the loss bands centered at $\lambda_{1}, \lambda_{2}$ and $\lambda_{3}$.

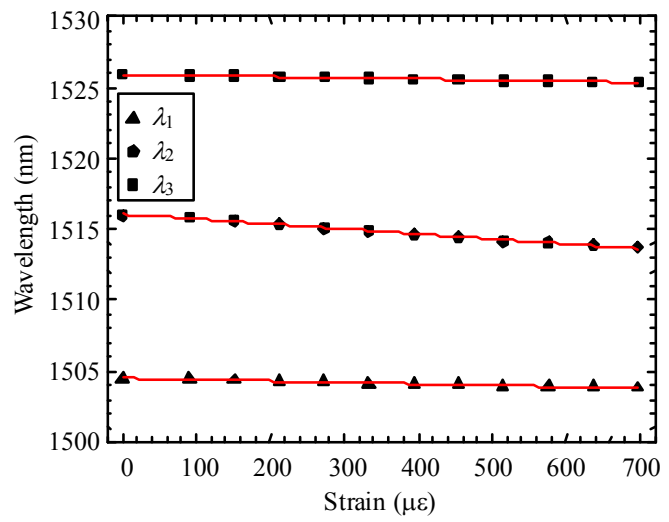

Fig. 5 Strain response of the sensing head for the loss bands centered at $\lambda_{1}, \lambda_{2}$ and $\lambda_{3}$.

The sensing structure was also characterized in terms of strain. The input and output fibers were accordingly fixed at two points that were $330 \mathrm{~mm}$ apart and submitted to specific strain values by using a translation stage (via sequential $50-\mu \mathrm{m}$ displacements). The results are made available in Fig. 5. The resonances had linear responses to strain variations, with different sensitivities: $(-1.02 \pm 0.04) \mathrm{pm} / \mu \varepsilon,(-3.51 \pm 0.01) \mathrm{pm} / \mu \varepsilon$ and $(-0.82 \pm 0.01) \mathrm{pm} / \mu \varepsilon$, for $\lambda_{1}, \lambda_{2}$ and $\lambda_{3}$, respectively. In this case, the fiber dimension decreased with increasing tensile stress thus changing the launching conditions between the single mode fiber and the step-index multimode D-shape fiber. Therefore, the Poisson coefficient was the dominant factor for the spectral deviation from shorter wavelengths. The 
different sensitivities resulted from the lack of symmetry of the D-shape fiber, and the refractive index change due to the variation of the fiber dimension was strongly wavelength dependent.

The sensitivity coefficients obtained for the refractive index, temperature, and strain applied to the proposed sensing structure indicated the feasibility to perform simultaneous measurement of these measurands by pairs [(temperature, refractive index) and (temperature, strain)].

\section{Conclusions}

An interferometer based on a D-shape chaotic optical fiber for measurement of multiparameters, was demonstrated. The sensing structure relied on a D-shape fiber section spliced in between two single mode fibers and interrogated in transmission. The optical spectrum presented multiple interference loss peaks as the result of multiple wave interference between a large number of different modes, which proved to be wavelength-sensitive to the refractive index, temperature and strain: maximum sensitivities of $95.2 \mathrm{~nm} / \mathrm{RIU}, 10.5 \mathrm{pm} /{ }^{\circ} \mathrm{C}$ and $-3.51 \mathrm{pm} / \mu \varepsilon$, respectively, could be achieved. The attained results made feasible the utilization of the proposed sensing structure for simultaneous measurement of parameter pairs [(temperature, refractive index) or (temperature, strain)].

Open Access This article is distributed under the terms of the Creative Commons Attribution License which permits any use, distribution, and reproduction in any medium, provided the original author(s) and source are credited.

\section{References}

[1] M. Corke, A. D. Kersey, D. A. Jackson, and J. D. C. Jones, "All-fiber Michelson thermometer," Electronics Letters, vol. 19, no. 13, pp. 471-473, 1983.

[2] J. A. Bucaro, H. D. Dardy, and E. F. Carome, "Fiber optic hydrophone," Journal of the Acoustical Society of America, vol. 62, no. 5, pp. 1302-1304, 1977.

[3] P. L Swart, "Long-period grating Michelson refractometric sensor," Measurement Science Technology, vol. 15, no. 8, pp. 1576-1580, 2004.

[4] A. D. Kersey, D. A. Jackson, and M. Corke, "A simple fiber Fabry-Perot sensor," Optics Communications, vol. 45, no. 2, pp. 71-74, 1983.

[5] J. Zhang, Y. Zhang, W. Sun, and L. Yuan, "Multiplexing multimode fiber and Fizeau etalon a simultaneous measurement scheme of temperature and strain," Measurement Science Technology, vol. 20, no. 6 , pp. $065206,2009$.

[6] F. Farahi, D. J. Webb, J. D. C. Jones, and D. A. Jackson, "Simultaneous measurement of temperature and strain: cross-sensitivity considerations," Journal Lightwave Technology, vol. 8, no. 2, pp. 138-142, 1990.

[7] V. Doya, O. Legrand, F. Mortessagne, and C. Miniatura, "Speckle statistics in a chaotic multimode fiber," Physical Review E, vol. 65, no. 5, pp. 056223-1-056223-15, 2002.

[8] V. Doya, O. Legrand, C. Michel, and F. Mortessagne, "Optical scar in a chaotic fiber," European Physical Journal-Special Topics, vol. 145, no. 1, pp. 49-61, 2007.

[9] P. Leproux, S. Février, V. Doya, P. Roy, and D. Pagnoux, "Modeling and optimization of double-clad fiber amplifiers using chaotic propagation of the pump," Optical Fiber Technology, vol. 7, no. 4 pp. 324-339, 2001.

[10] P. Leproux, V. Doya, P. Roy, D. Pagnoux, F. Mortessagne, and O. Legrand, "Experimental study of pump power absorption along rare-earth-doped double clad optical fibers," Optics Communication, vol. 218, no. 4-6, pp. 249-254, 2003.

[11] V. Doya, O. Legrand, F. Mortessagne, and C. Miniatura, "Light scarring in an optical fiber," Physical Review Letters, vol. 88, no. 1, pp. 014102, 2002.

[12] E. Li, "Temperature compensation of multimode-interference-based fiber devices," Optics Letters, vol. 32, no. 14, pp. 2064-2066, 2007. 